\title{
Subtyping Patients with Myalgic Encephalomyelitis (ME) and Chronic Fatigue Syndrome (CFS) By Course of Illness
}

\author{
Jamie Stoothoff* , Kristen Gleason, Stephanie McManimen, Taylor Thorpe and Leonard A. Jason
}

Center for Community Research, DePaul University, Chicago, IL, USA

Received: March 22,2017; Accepted: June 08,2017; Published:June 26,2017

*Corresponding authors: Jamie Stoothoff, Center for Community Research, DePaul University 990 W. Fullerton Ave., Suite 3100 Chicago, IL, USA 60604-2201, Tel.: (773) 325-2018;Fax: (773) 325-4923,E-mail: jstootho@depaul.edu

\begin{abstract}
Past research has subtyped patients with Myalgic Encephalomyelitis (ME) and Chronic Fatigue Syndrome (CFS) according to factors related to illness onset, illness duration, and age. However, no classification system fully accounts for the wide range of symptom severity, functional disability, progression, and prognosis seen among patients. This study examined whether illness trajectories among individuals with CFS were predictive of different levels of symptomology, functional disability, and energy expenditure. Of the participants $(N=541)$, the majority described their illness as Fluctuating (59.7\%), with 15.9\% Constantly Getting Worse, 14.1\% Persisting, 8.5\% Relapsing and Remitting, and 1.9\% Constantly Getting Better. The illness courses were associated with significant differences in symptomology on select domains of the DSQ functioning on select subscales of the SF-36, and on overall levels of energy expenditure. The significant symptomatic and functional differences between groups suggest that subtyping patients with CFS according to illness course is a promising method for creating more homogeneous groups of patients.
\end{abstract}

Keywords: Myalgic Encephalomyelitis; Chronic Fatigue Syndrome; Subtypes; Illness Course;

\section{Introduction}

\section{Subtyping Patients with Myalgic Encephalomyelitis (ME) and Chronic Fatigue Syndrome (CFS) by Course of Illness}

Myalgic Encephalomyelitis (ME) and Chronic Fatigue Syndrome (CFS) are debilitating illnesses, often presenting with neurological, immunological, gastrointestinal, musculoskeletal and hormonal symptoms [1-4]. The severity of symptoms and levels of functional disability experienced can vary drastically from patient to patient, suggesting there may be distinct clinical courses that have yet to be identified. In the most extreme cases, patients are bedbound and depend entirely on caretakers for support. Community based studies have determined that approximately $25 \%$ of patients are housebound and $64.3 \%$ are on disability, with only $15.1 \%$ of patients currently employed [5-7]. In short, those diagnosed with these illnesses represent a heterogeneous group of individuals who experience differing patterns of symptom severity and levels of functional disability [8].

It is possible that combining potentially diverse patient subtypes into one sample pool may account for the inconsistent findings and the lack of reproducibility that has often plagued research related to the etiology, diagnosis, epidemiology, and treatment of ME and CFS [8]. Past studies have attempted to categorize patients based on type of illness onset [9-11], the duration of the illness, and the age of patient [12-15]. However, there is no classification system yet that fully accounts for the diversity of symptom severity, functional disability, progression, and prognosis of this illness. It is, therefore, pertinent to further explore methods of subtyping individuals with ME and CFS in order to more accurately describe the wide range of patient experiences.

Research has distinguished between two different onset patterns: 1) sudden onset, in which symptoms appear over a few days or weeks and 2) gradual onset, in which symptoms develop over a period of months or years. Sudden onset has often been linked with viral and infectious illnesses [16], including: EpsteinBarr Virus infection (EBV) and enterovirus infections $[17,18]$. One study found that patients who experienced a sudden onset had poorer health outcomes than patients who described their onset as gradual [9]. Conversely, other findings suggest that patients with a sudden onset have a better prognosis than those with a gradual onset, while others have found no significant differences in prognosis between sudden and gradual onset subgroups [10-12]. With such a range of mixed results, subtyping patients by type of onset cannot fully account for the variability seen in symptom severity and the course of the illness.

Additionally, researchers have explored illness duration and current age as related factors that may impact prognosis and symptom severity. Research has found that patients with a longer illness duration (10 years or more) experienced poorer cognitive functioning compared to those with a shorter illness 
duration (7 years or less) [13]. However, another study has found that the physical functioning of patients tended to improve over the course of their illness [14]. Further studies were unable to reproduce these findings and have suggested that the length of the illness experience does not have a significant effect on patient outcomes [12]. In general, older age has also predicted poorer prognosis among patients [15]. In one study, researchers sought to examine how both age and duration of illness can interact to affect illness severity and functioning. Within this sample, older patients with longer illness duration (10 years or more) displayed better mental health functioning than younger patients and older patients with shorter illness duration. However, younger patients with an illness duration of 10 years or more reported more severe immune and autonomic symptoms than older patients whose illness experience also spanned 10 years or more [16].

The categorization system developed to describe differing clinical courses in patients with Multiple Sclerosis (MS) is an illustrative example of the importance of defining subtypes of a chronic illness. The International Advisory Committee on Clinical Trials of MS has defined four distinct subtypes of illness course: clinically isolated syndrome, relapsing-remitting, primary progressive and secondary progressive. Clinically isolated syndrome describes patients who have experienced their first episode of neurological symptoms. The relapsing-remitting course is characterized by attacks of new neurologic symptoms, followed by periods of partial or complete recovery, while primary progressive describes patients who have experienced consistently worsening neurologic functioning without relapses or remissions. The final subtype, secondary progressive, describes a worsening of neurological symptoms over time, following a relapsing and remitting course [20].

Using the MS typology described above as a template, the current study explored the feasibility of a similar system of classifying differing experiences of CFS and ME symptomology. Specifically, we examined whether differing self-reported patient illness courses are predictive of different levels of symptom severity and functional disability. Further, we assessed the relationship between these illness course subtypes, maintenance of the energy envelope, illness duration, and age.

\section{Methods}

\section{Research Participants}

The sample for the present study was derived from multiple settings in various geographical locations. Participants included individuals at least 18 years of age with a current diagnosis of CFS or ME. Participants from the DePaul sample had self-report diagnoses, while the remaining samples required diagnoses to be confirmed by a physician. Of the 882 individuals available, only participants with complete data on the primary illness course variable $(N=541)$ were included.

The overall sample was $84.5 \%$ female and $15.5 \%$ male. Most participants identified as Caucasian/White (98.2\%); 0.4\% were Asian or Pacific Islander, and $1.4 \%$ indicated their race as "other." For current work status, $62.5 \%$ reported being on disability, $17.3 \%$ reported they were working, and the remaining 20.2\% were not currently employed (student, homemaker, retired, or unemployed). Most participants in this sample $(55.8 \%)$ were married or living with someone; $29.1 \%$ were single and $15.1 \%$ were either divorced, separated, or widowed. In terms of education, $30.4 \%$ of participants had a high school diploma or lower level of education, $11.6 \%$ attended college for at least a year, $33.6 \%$ held a standard college degree, and $24.4 \%$ had a graduate degree. The mean age was 46.3 years $(S D=13.2)$.

\section{DePaul Sample}

The largest portion of participants for the present study ( $n=213,39.4 \%$ ) came from the DePaul convenience sample pool. This sample was recruited through support groups, internet forums, and by contacting past participants of DePaul studies. Participants were English speakers at least 18 years old who self-identified as having a CFS or ME diagnosis. Those eligible completed informed consents and surveys either electronically, by hard-copy, or verbally over the phone. A large majority of the DePaul participants were female $(84.2 \%$ versus $15.8 \%$ male $)$ and most identified as Caucasian/White (97.7\%). An additional $0.4 \%$ identified as Asian and 1.9\% reported their race as "other." The mean age for the DePaul Sample group was 51.9 (SD = 11.3).

\section{Newcastle Sample}

Another $18.1 \%$ of the participants $(n=98)$ in the present study came from the Newcastle Sample. Individuals 18 years of age and older with a suspected CFS diagnosis were referred to the Newcastle-upon-Tyne Royal Infirmary clinic for a medical assessment. A physician completed a comprehensive medical history and medical examination for each participant. All informed consents and measures were completed by hard copy. Most of the Newcastle Sample group (84.2\%) identified as female and $15.8 \%$ identified as male. Almost all participants from this group identified as Caucasian (99.0\%), and 1 participant selected "other" as their primary race. The mean age was 45.7 years (SD = 14.0).

\section{Norway 1 Sample}

The Norway 1 Sample database contributed $34.4 \%$ of the participants in the present study $(\mathrm{n}=168)$. Participants living in the suburbs of Oslo, Norway, were recruited to participate in a CFS self-management trial program, through referrals from healthcare professions as well as through CFS patient organizations and education programs. Patients were required to be 18 years of age or older, have a current CFS diagnosis, and be physically able to attend the self-management program. Participants completed an informed consent that included permission to request a confirmation of their CFS diagnosis. Within this sample of 168 individuals, $87.4 \%$ were female and $12.6 \%$ were male. Nearly all participants identified as Caucasian (99.4\%), and 1 participant identified as "other". The mean age was 43.4 years $(S D=11.6)$.

\section{Norway 2 Sample}

The final $11.5 \%$ of the sample in the present study 
came from the Norway 2 Sample database. Participants from this database were recruited from an inpatient medical facility and an outpatient CFS/ME Center. Participants were required to be between 18 and 65 years of age. An experienced physician completed a comprehensive medical history interview and medical examination for individuals suspected of having a CFS diagnosis. In addition, a psychologist examined each individual to rule out exclusionary conditions. All informed consent and other study measures were completed by hard copy. Again, the majority of participants were female (82.3\%) and $17.7 \%$ were male. Regarding race, $95.0 \%$ identified as Caucasian, $1.7 \%$ as Asian and $3.3 \%$ identified as "other." The mean age was 34.8 years $(S D=$ 11.7).

\section{Illness Course Groups}

Participants were placed in one of five illness course groups based upon their response to an item asking about the progression of their illness. This item provided the choice of five labels: "constantly getting worse,"'constantly improving," "persisting (no change),"“relapsing and remitting (having 'good' periods with no symptoms alternating with symptomatically 'bad' periods)," and "fluctuating (symptoms periodically get better and get worse, but never disappear completely)."

\section{Measures}

\section{SF-36}

Participants completed the Medical Outcomes Study 36-item short-form health survey (SF-36) to measure their current physical and mental functioning [21]. The questionnaire measures functioning on eight subscales: Physical Functioning, Role Physical, Bodily Pain, General Health, Social Functioning, Mental Health, Role Emotional, and Vitality. This measure is scored on a 100-point scale, where higher scores indicate higher functioning, and lower scores indicate lower functioning. The SF-36 has been found to have good internal consistency and discriminate validity among subscales [22].

\section{DePaul Symptom Questionnaire (DSQ)}

The DePaul Symptom Questionnaire (DSQ) $)^{[7]}$ consists of 54 self-report items assessing CFS-related symptomatology, as well as demographic, occupational, and social history. Patients were asked to rate the frequency of their symptoms over the past 6 months on a 5-pt Likert scale as follows: $0=$ none of the time, 1 = a little of the time, 2 = about half the time, $3=$ most of the time, and $4=$ all of their time. Patients were also asked to rate the severity of their symptoms over the past 6 months on a 5-pt Likert scale as follows: $0=$ symptom not present, $1=$ mild, 2 = moderate, 3 = severe, and 4 = very severe. Symptom frequency and severity were converted to a 100-point scale, and were averaged to create one composite score for each symptom. Symptoms were compiled into domains ${ }^{[23]}$ based on symptomology suggested by the Canadian Clinical Criteria for $\mathrm{ME} / \mathrm{CFS}^{[2]}$ and select other symptoms categories that were empirically identified through factor analysis [23,2,7]. The domain composite scores were calculated by converting the frequency and severity for each symptom in the domain and averaging them to create one score. The DSQ has proven to have good test-retest reliability, as well as good convergent and discriminate validity $[24,25]$. The DSQ has been made available at Research Electronic Data Capture (REDCap): https://redcap. is.depaul.edu/surveys/?s=tRxytSPVVw.

\section{Energy Quotient}

Energy quotients were calculated from information provided on the DSQ and used to assess how much energy participants were expending in comparison to how much they had available. The Energy Envelope theory suggests that patients who monitor their activity and are mindful not to overexert themselves will experience better health outcomes and fewer symptoms such as Post-Exertional Malaise (PEM) [26]. Using a Likert scale ranging from one (no energy) to 100 (pre-illness energy level), participants were asked to rate their available and expended energy yesterday and last week. To calculate energy quotients for both yesterday and last week, expended energy levels were divided by available energy levels and then multiplied by 100 . Scores over 100 indicate overextension and scores under 100 indicate under extension. Due to multiple outliers, a winsorizing technique was used to minimize their effect. Energy scores within the top $5^{\text {th }}$ percentile were replaced with the value corresponding to the $95^{\text {th }}$ percentile.

\section{Statistical Analysis}

A series of one-way ANOVAs were used to compare mean differences between illness subtypes for the following categories: SF-36 subscales, DSQ symptom items, illness duration, age, and energy quotients. Missing data were handled using pair wise deletion and all analyses were performed using SPSS software (version 21). The Games-Howell post-hoc test was used to examine significant differences between groups.

\section{Results}

\section{Illness Course Groups}

Most the sample (59.7\%), described the course of their illness as Fluctuating, with symptoms periodically alternating between getting better and getting worse but never disappearing completely. Constantly Getting Worse comprised the second largest group, representing $15.9 \%$ of the sample. Similarly, $14.1 \%$ of the sample described their illness as Persisting, which was characterized by having no changes in symptomatology. The Relapsing and Remitting group represented $8.5 \%$ of the total sample, and was defined by having "good" periods with no symptoms alternating with symptomatically "bad" periods. The remaining $1.9 \%$ of the sample described their illness as Constantly Getting Better.

\section{Demographics}

Table 1 shows the demographic information for each illness course subtype. A series of chi-square tests were performed and no significant relationship was found between illness course groups and gender, race, ethnicity, marital status, education, or work status. In addition, a series of one-way ANOVAs determined 


\begin{tabular}{|c|c|c|c|c|c|}
\hline & $\begin{array}{c}\text { Constantly } \\
\text { Improving }(\mathrm{N}=10)\end{array}$ & $\begin{array}{l}\text { Relapsing \& Remitting } \\
\qquad(\mathrm{N}=46)\end{array}$ & Fluctuating $(\mathrm{N}=323)$ & Persisting (N=76) & $\begin{array}{c}\text { Constantly Getting } \\
\text { Worse (N=86) }\end{array}$ \\
\hline & $\mathrm{M}$ (SD) & $\mathrm{M}$ (SD) & $\mathrm{M} \quad(\mathrm{SD})$ & M $(S D)$ & $\mathrm{M} \quad(\mathrm{SD})$ \\
\hline $\begin{array}{l}\text { Age } \\
\text { Age of Diagnosis } \\
\text { Duration of Illness }\end{array}$ & $\begin{array}{l}42.55(18.02) \\
36.30(15.01) \\
13.10(10.02)\end{array}$ & $\begin{array}{c}46.11(13.87) \\
35.51(12.49) \\
8.03(6.78)\end{array}$ & $\begin{aligned} 45.29 & (12.84) \\
37.93 & (11.66) \\
8.64 & (7.27)\end{aligned}$ & $\begin{array}{ll}47.58 & (13.78) \\
38.84 & (10.94) \\
10.05 & (8.47)\end{array}$ & $\begin{array}{c}48.85(12.67) \\
39.84(11.70) \\
9.70(7.74)\end{array}$ \\
\hline Duration of Illness & $\%(\mathrm{~N})$ & $\%(\mathrm{~N})$ & $\%(\mathrm{~N})$ & $\%(\mathrm{~N})$ & $\%(\mathrm{~N})$ \\
\hline \multicolumn{6}{|l|}{ Gender } \\
\hline $\begin{array}{l}\text { Male } \\
\text { Female }\end{array}$ & $\begin{array}{ll}20.00 & (2) \\
80.00 & (8)\end{array}$ & $\begin{array}{c}8.76(4) \\
91.30(42)\end{array}$ & $\begin{array}{ll}14.33 & (46) \\
85.67 & (275)\end{array}$ & $\begin{array}{l}22.37(17) \\
77.63(59)\end{array}$ & $\begin{array}{l}17.44(15) \\
82.56(71)\end{array}$ \\
\hline \multicolumn{6}{|l|}{ Race } \\
\hline $\begin{array}{l}\text { White } \\
\text { Asian or Pacific Islander } \\
\text { Other }\end{array}$ & $\begin{aligned} 100.00 & (10) \\
0.00 & (0) \\
0.00 & (0)\end{aligned}$ & $\begin{aligned} 100.00 & (45) \\
0.00 & (0) \\
0.00 & (0)\end{aligned}$ & $\begin{aligned} 97.52 & (314) \\
0.62 & (2) \\
1.86 & (6)\end{aligned}$ & $\begin{aligned} 100.00 & (75) \\
0.00 & (0) \\
0.00 & (0)\end{aligned}$ & $\begin{array}{ll}97.67 & (84) \\
0.00 & (0) \\
2.32 & (2)\end{array}$ \\
\hline \multicolumn{6}{|l|}{ Ethnicity } \\
\hline $\begin{array}{l}\text { Non-Hispanic } \\
\text { Hispanic }\end{array}$ & $\begin{aligned} 100.00 & (10) \\
0.00 & (0)\end{aligned}$ & $\begin{aligned} 100.0 & (45) \\
0.00 & (0)\end{aligned}$ & $\begin{aligned} 98.41 & (310) \\
1.59 & (5)\end{aligned}$ & $\begin{aligned} 100.00 & (73) \\
0.00 & (0)\end{aligned}$ & $\begin{aligned} 98.80 & (83) \\
1.20 & (1)\end{aligned}$ \\
\hline \multicolumn{6}{|l|}{ Marital Status } \\
\hline $\begin{array}{l}\text { Married } \\
\text { Separated } \\
\text { Widowed } \\
\text { Divorced } \\
\text { Never Married }\end{array}$ & $\begin{array}{r}22.22(2) \\
0.00(0) \\
0.00(0) \\
22.22(2) \\
55.56(5)\end{array}$ & $\begin{aligned} 59.09 & (26) \\
2.27 & (1) \\
0.00 & (0) \\
9.09 & (4) \\
29.55 & (13)\end{aligned}$ & $\begin{aligned} 58.31 & (186) \\
1.25 & (4) \\
0.94 & (3) \\
12.54 & (40) \\
6.96 & (86)\end{aligned}$ & $\begin{aligned} 54.05 & (40) \\
1.35 & (1) \\
0.00 & (0) \\
13.51 & (10) \\
31.08 & (23)\end{aligned}$ & $\begin{aligned} 50.00 & (43) \\
2.32 & (2) \\
0.00 & (0) \\
15.11 & (13) \\
32.56 & (28)\end{aligned}$ \\
\hline \multicolumn{6}{|l|}{ Education } \\
\hline $\begin{array}{l}\text { High School } \\
\text { Partial College } \\
\text { College Degree } \\
\text { Graduate Degree }\end{array}$ & $\begin{array}{ll}40.00 & (4) \\
20.00 & (2) \\
20.00 & (2) \\
20.00 & (2)\end{array}$ & $\begin{array}{ll}39.13 & (18) \\
13.04 & (6) \\
26.09 & (12) \\
21.74 & (10)\end{array}$ & $\begin{array}{ll}27.67 & (88) \\
12.26 & (39) \\
35.53 & (113) \\
24.53 & (78)\end{array}$ & $\begin{array}{ll}31.51 & (23) \\
10.96 & (8) \\
28.77 & (21) \\
28.77 & (21)\end{array}$ & $\begin{array}{c}34.94(29) \\
7.23(6) \\
37.35(31) \\
20.48(17)\end{array}$ \\
\hline \multicolumn{6}{|l|}{ Work Status } \\
\hline $\begin{array}{l}\text { On Disability } \\
\text { Retired } \\
\text { Working } \\
\text { Not Working }\end{array}$ & $\begin{array}{ll}40.00 & (4) \\
10.00 & (1) \\
20.00 & (2) \\
30.00 & (3)\end{array}$ & $\begin{aligned} 47.83 & (22) \\
6.52 & (3) \\
30.43 & (14) \\
15.22 & (7)\end{aligned}$ & $\begin{aligned} 62.42 & (201) \\
7.76 & (25) \\
17.39 & (56) \\
12.42 & (40)\end{aligned}$ & $\begin{aligned} 69.33 & (52) \\
13.33 & (10) \\
12.00 & (9) \\
5.33 & (4)\end{aligned}$ & $\begin{aligned} 67.44 & (58) \\
8.17 & (7) \\
13.95 & (12) \\
10.47 & (9)\end{aligned}$ \\
\hline
\end{tabular}

the groups did not differ significantly in age at time of self-report, age at diagnosis, or illness duration.

\section{DSQ Domain Differences}

Table 2 shows the means for DSQ domain composites for each illness course group, along with the total sample. There were significant differences in symptom scores between groups for the following domains: the Immune Domain $(F(4,535)=5.3, p=.000)$, the Pain Domain $(F(4,535)=6.8, p=.000)$, the Post-Exertional Malaise Domain, $F(4,535)=18.7, \mathrm{p}=.00$, the Sleep Domain $(\mathrm{F}(4$,
$535)=5.1, \mathrm{p}=.002)$, the Gastrointestinal Domain $(\mathrm{F}(4,535)=$ $4.6, \mathrm{p}=.001)$ and the Orthostatic Intolerance Domain $(\mathrm{F}(4,535)$ $=4.6, \mathrm{p}=.001$ ). The Constantly Getting Worse group generally reported higher levels of symptom severity and frequency when compared to the other groups, while the Constantly Improving group reported lower levels of symptom severity and frequency compared to the other groups. The remaining three groups had intermediate scores on these domains. 


\begin{tabular}{|c|c|c|c|c|c|c|c|}
\hline & $\begin{array}{l}\text { Constantly } \\
\text { Improving }\end{array}$ & $\begin{array}{c}\text { Relapsing \& } \\
\text { Remitting }\end{array}$ & Fluctuating & Persisting & $\begin{array}{c}\text { Constantly } \\
\text { Getting Worse }\end{array}$ & & $\begin{array}{l}\text { Total } \\
\text { sample }\end{array}$ \\
\hline Post-Exertional Malaise & 34.58 abc & 61.10 de & 68.82 af & 74.72 bd & 79.55 cef & $* *$ & 70.05 \\
\hline Minimum exercise makes tired & $42.50^{\text {ab }}$ & $67.77^{c}$ & $74.90^{\mathrm{d}}$ & $80.50^{\text {a }}$ & 85.73 bcd & $* *$ & 76.21 \\
\hline Drained/sick after mild activity & 35.00 ab & $62.77^{\mathrm{cd}}$ & 70.52 ef & 78.78 ace & 83.38 bdf & $* *$ & 72.40 \\
\hline Soreness after mild activity & 27.50 abcd & 67.93 ae & 70.80 bf & $77.30^{c}$ & 81.47 def & $* *$ & 72.35 \\
\hline Dead/heavy feeling after exercise & $35.00 \mathrm{ab}$ & $59.88^{\mathrm{cd}}$ & $71.15^{\text {e }}$ & 76.73 ac & 83.17 bde & $* *$ & 72.13 \\
\hline Mentally tired after slightest effort & 31.25 abc & $56.52 \mathrm{~d}$ & 62.30 ae & 69.57 b & 72.91 cde & $* *$ & 63.92 \\
\hline Muscle weakness & 36.25 a & 52.17 b & 62.46 & 64.53 & 71.17 ab & $* *$ & 62.76 \\
\hline Sleep & 45.50 & $57.41^{\mathrm{a}}$ & $59.66^{b}$ & 62.15 & 68.00 ab & $* *$ & 60.87 \\
\hline Unrefreshing sleep & 58.75 a & $73.33 \mathrm{~b}$ & 81.38 & 84.70 & 86.76 ab & $* *$ & 81.61 \\
\hline Problems staying asleep & 46.25 & 59.37 & 59.09 & 60.16 & 67.48 & & 60.34 \\
\hline Problems falling asleep & 53.75 & 52.98 & 57.76 & 58.71 & 66.32 & & 58.78 \\
\hline Need to nap daily & $26.25^{a b c}$ & $46.94^{\mathrm{d}}$ & 55.39 ae & $60.03 \mathrm{~b}$ & 67.24 cde & $* *$ & 56.64 \\
\hline Waking up early & 42.50 & 50.85 & 43.63 & 46.60 & 51.93 & & 45.94 \\
\hline Neurocognitive & 47.85 & 59.37 & $60.69^{a}$ & 65.35 & $68.66^{a}$ & $* *$ & 62.25 \\
\hline Difficulty paying attention & 48.75 & 69.70 & 71.79 a & 78.94 & $81.54^{a}$ & $* *$ & 73.73 \\
\hline Problems remembering & 53.75 & 65.21 & 66.73 & 72.58 & 74.32 & $*$ & 68.35 \\
\hline Can only focus on one thing at a time & 48.75 & 61.41 & 62.07 a & 68.75 & $74.60^{a}$ & $* *$ & 64.66 \\
\hline Difficulty expressing thoughts & 56.25 & 62.22 & 60.68 & 67.51 & 68.01 & & 62.85 \\
\hline Absent-mindedness & 52.50 & 56.66 & 59.86 & 61.75 & 66.90 & & 60.84 \\
\hline Difficulty understanding & 33.75 & 45.38 & 46.09 & 50.00 & 54.46 & $*$ & 47.67 \\
\hline Immune & 14.47 abcd & $32.31^{a}$ & $37.90^{b}$ & $37.59^{c}$ & $42.17 \mathrm{~d}$ & $* *$ & 37.63 \\
\hline Flu-like symptoms & 21.25 abcd & $48.36^{a}$ & 54.90 b & $55.75^{c}$ & $60.88^{d}$ & $* *$ & 54.79 \\
\hline Sore throat & 9.72 abcd & $33.33^{a}$ & 38.97 b & $38.00^{d}$ & $42.57 \mathrm{~d}$ & $* *$ & 38.44 \\
\hline Tender lymph nodes & 16.25 a & 31.39 & 36.55 & 40.41 a & 40.44 & $*$ & 36.91 \\
\hline Fever & 10.00 & 16.19 & 20.55 & 12.86 & 24.09 & $*$ & 19.75 \\
\hline Neuroendocrine /Circulatory & 38.43 & 42.54 & 47.91 & 42.34 & 49.04 & & 46.76 \\
\hline Cold limbs & 47.50 & 49.72 & 56.90 & 55.66 & 55.88 & & 55.79 \\
\hline Feeling hot/cold for no reason & 52.50 & 53.53 & 56.25 & 51.43 & 57.13 & & 55.42 \\
\hline Chills/shivers & 38.75 & 41.57 & 45.47 & 35.44 & 47.50 & & 43.92 \\
\hline Low temperature & 15.00 & 29.34 & 33.00 & 26.50 & 35.86 & & 31.89 \\
\hline Pain & 32.50 abc & $49.72 d$ & $61.99^{a}$ & 63.48 b & $69.26 \mathrm{~cd}$ & $* *$ & 61.76 \\
\hline Muscle pain & $32.50 \mathrm{abc}$ & 52.77 de & $64.94^{a}$ & 68.91 bd & 73.52 се & $* *$ & 65.24 \\
\hline Joint pain & 32.50 a & 47.82 b & 58.67 & 58.50 & $65.00 \mathrm{ab}$ & $* *$ & 58.23 \\
\hline Gastrointestinal & $23.33^{a}$ & 45.08 & 47.90 & $40.23^{b}$ & 52.89 ab & $* *$ & 46.91 \\
\hline Irritable bowel problems & 25.00 & 50.00 & 50.85 & $40.78^{a}$ & $59.37 \quad$ a & $* *$ & 50.21 \\
\hline Bloating & 21.25 & 46.19 & 49.02 & 42.26 & 52.25 & $* *$ & 47.81 \\
\hline Stomach pain & 23.75 & 38.85 & 43.88 & 37.58 & 46.91 & $*$ & 42.67 \\
\hline
\end{tabular}




\begin{tabular}{|c|c|c|c|c|c|c|c|}
\hline Orthostatic Intolerance & $18.00{ }^{a}$ & 35.08 & 37.29 & 34.31 & $42.41{ }^{a}$ & ** & 37.14 \\
\hline Unsteady on feet & 20.00 & 41.84 & 42.89 & 43.83 & 43.52 & & 42.61 \\
\hline Dizziness/fainting & 20.00 & 37.77 & 42.00 & 42.43 & 45.29 & & 41.81 \\
\hline Shortness of breath & $17.50^{\text {a }}$ & 37.50 & 39.90 & $34.86^{\mathrm{b}}$ & $48.67 \mathrm{ab}$ & ** & 39.97 \\
\hline Irregular heart beats & $17.50^{\text {a }}$ & 30.27 & 34.00 & $26.66^{b}$ & $41.86^{a b}$ & ** & 33.57 \\
\hline Chest pain & 15.00 & 27.17 & 27.17 & 23.32 & 32.79 & & 27.29 \\
\hline
\end{tabular}

\section{SF-36 Subscale Scores}

Table 3 shows the means and standard deviations for each SF-36 subscale across each illness course subtype, as well as for the entire sample. There were significant differences in functioning scores between groups for the following subscales:Role Physical $(\mathrm{F}(4,519)=18.7, \mathrm{p}=.000)$, Bodily Pain
$(\mathrm{F}(4,519)=9.01, \mathrm{p}=.000)$, Physical Functioning $(\mathrm{F}(4,520)=$ $10.01, \mathrm{p}=.000)$,Vitality $\mathrm{F}(4,520)=11.4, \mathrm{p}=.000)$, General Health $(\mathrm{F}(4,515)=4.5, \mathrm{p}=.001$.$) , and Mental Health (\mathrm{F}(4,519)=2.7, \mathrm{p}$ $=.03$ ). The Constantly Getting Worse group reported significantly worse functioning on all subscales except the General Health and the Role Emotional subscales.

Table 3. ANOVA results for mean (SD) differences on SF-36 subscales between illness subtypes

\begin{tabular}{|c|c|c|c|c|c|c|c|}
\hline & $\begin{array}{l}\text { Constantly } \\
\text { Improving }\end{array}$ & $\begin{array}{l}\text { Relapsing \& } \\
\text { Remitting }\end{array}$ & Fluctuating & Persisting & $\begin{array}{c}\text { Constantly Getting } \\
\text { Worse }\end{array}$ & & Total Sample \\
\hline & $\mathrm{M}$ (SD) & $M \quad(S D)$ & $M \quad(S D)$ & $\mathrm{M}$ (SD) & $M \quad(S D)$ & & $M \quad(S D)$ \\
\hline Role Physical & $52.50(29.93){ }^{a b c d}$ & $5.36(17.92) \quad$ a & $6.53(18.88)^{b}$ & $3.89(11.73)^{c}$ & $4.22(11.59){ }^{d}$ & ** & $6.57(18.36)$ \\
\hline Vitality & 40.00 (27.39) & $24.38(15.16)$ ab & $19.19(15.61)^{\mathrm{cd}}$ & $12.65(15.08)^{a c}$ & 13.25 (14.49) bd & $* *$ & $18.12(16.21)$ \\
\hline Social Functioning & $53.75(40.41)$ & $36.34(26.70)$ ab & $28.30(22.49)^{c}$ & $22.20(23.24)^{a}$ & $14.43(18.15) b c$ & $* *$ & 26.35 (23.76) \\
\hline General Health & $43.80(19.84)$ & $33.05(14.23)^{a}$ & $28.30(16.65)$ & $25.16(15.00)^{a}$ & 25.05 (18.29) & $* *$ & $28.03(16.80)$ \\
\hline Physical Functioning & $58.83(36.70)$ & $44.81(22.94)^{a b}$ & $38.01(22.31)^{c}$ & $31.64(20.63)^{a}$ & $25.89(20.98)$ bc & ** & $36.10(23.01)$ \\
\hline Bodily Pain & $70.40(21.77)$ & $48.56(23.58)^{d}$ & $39.39(22.02)$ ae & $37.72(25.96)^{b}$ & $30.96(24.66)$ cde & $* *$ & 39.14 (23.89) \\
\hline Mental Health & $77.60(15.57) a b c$ & $77.67(13.78)^{a b}$ & $69.24(18.06)^{a}$ & 69.66 (20.65) & $68.56(18.05)^{b}$ & $*$ & 70.04 (18.23) \\
\hline Role Emotional & 93.33 (21.08) & 79.07 (37.81) & $72.69(40.75)$ & 76.58 (39.68) & 80.32 (37.19) & & $75.38(39.40)$ \\
\hline \multicolumn{8}{|c|}{$\begin{array}{l}\text { Similar letters denote significant differences } \\
{ }^{*} \mathrm{p}<.05 \\
{ }^{* *} \mathrm{p}<.01\end{array}$} \\
\hline
\end{tabular}

\section{Energy Envelope}

Table 4 displays the means and standard deviations on reported levels of fatigue and energy for the five subtypes, as well as for the entire sample. There were significant differences between groups in Past Week Fatigue scores $(F(4,506)=11.60$, p $=.00)$ and Past Week Available Energy scores $(F(4,509)=14.55$, $\mathrm{p}=.00)$. The Constantly Getting Worse group and the Persisting group reported significantly more fatigue than the remaining three groups. There was also a significant difference for Past Week Energy Quotients between illness subtypes $(\mathrm{F}(4,497)=$ $6.01, \mathrm{p}=.00)$. Scores describing participants' fatigue and energy levels for yesterday are also shown in Table 4. 


\begin{tabular}{|c|c|c|c|c|c|c|c|}
\hline & $\begin{array}{l}\text { Constantly } \\
\text { Improving }\end{array}$ & $\begin{array}{l}\text { Relapsing \& } \\
\text { Remitting }\end{array}$ & Fluctuating & Persisting & $\begin{array}{c}\text { Constantly Getting } \\
\text { Worse }\end{array}$ & & Total Sample \\
\hline & $\mathrm{M}$ (SD) & M (SD) & M (SD) & M (SD) & $\mathrm{M}$ (SD) & & $\mathrm{M}$ (SD) \\
\hline \multicolumn{8}{|l|}{ Past week } \\
\hline Fatigue & $45.60(25.23)$ ab & $61.70(21.78)^{\mathrm{cd}}$ & $67.55(20.19)$ ef & $77.39(17.86)$ ace & 77.05 (19.97) bdf & $* *$ & 69.55 (20.91) \\
\hline Energy quotient & $121.76(55.03)$ & $119.73(49.34)$ & $109.03(42.46)^{a}$ & $122.39(54.35)$ & $139.19(70.75)^{a}$ & ** & $116.79 \quad(51.41)$ \\
\hline Available energy & $45.00(19.72) a b$ & $35.27(15.77)^{\mathrm{cd}}$ & $31.19(16.16)^{\text {ef }}$ & 23.81 (13.41) ace & 20.27 (14.17) bdf & $* *$ & $29.00 \quad(16.33)$ \\
\hline Expended energy & $55.00(20.68)^{a b}$ & $41.71(20.15)^{c d}$ & 33.69 (19.86) & $28.85(16.67)^{a c}$ & $29.67(21.15)$ bd & $* *$ & $33.50 \quad(20.15)$ \\
\hline \multicolumn{8}{|l|}{ Yesterday } \\
\hline Fatigue & $46.10(27.49)^{a}$ & $55.20(25.92) \mathrm{bc}$ & $66.38(22.20)^{d}$ & $76.15(17.95)$ acd & $73.39(24.56)^{b}$ & $* *$ & $67.51 \quad(23.28)$ \\
\hline Energy quotient & 120.00 & 113.52 (51.99) & $100.07(38.55)^{a}$ & $113.45(50.68)^{\mathrm{b}}$ & $139.73(61.82)^{\mathrm{ab}}$ & $* *$ & 109.49 \\
\hline Available energy & $46.50(19.44)^{\mathrm{ab}}$ & $32.44(16.87)^{\mathrm{cd}}$ & $27.86(16.40)^{\mathrm{e}}$ & $23.63(14.48)^{\mathrm{ac}}$ & 18.05 (12.53) bde & $* *$ & $26.51 \quad(16.40)$ \\
\hline Expended energy & $60.50(25.65)^{a b c}$ & 37.16 (24.50) & $28.19(18.95)^{a}$ & $27.13(19.10)^{b}$ & $27.00(24.78)^{c}$ & $* *$ & 29.24 (21.17) \\
\hline \multicolumn{8}{|c|}{$\begin{array}{l}\text { Similar letters denote significant differences } \\
{ }^{*} \mathrm{p}<.05\end{array}$} \\
\hline
\end{tabular}

\section{Discussion}

The present study explored factors associated with differing self-reported illness course experiences: Constantly Getting Worse, Constantly Improving, Persisting, Relapsing and Remitting, and Fluctuating. The findings suggest that this method of grouping patients is a promising way to examine subtypes of CFS with distinct patterns of symptomology and levels of functioning. The majority of participants in our sample (59.7\%) defined the course of their illness as Fluctuating, which was described as "symptoms periodically get better and get worse, but never disappear completely." In general, this group reported the third most severe scores related to their levels of symptomology, functioning, available energy, and fatigue. Overall, these results suggest that the majority of participants experienced a moderate level of illness severity when compared to other subtypes.

The Constantly Getting Worse group, representing $15.9 \%$ of the sample, demonstrated the most severe and frequent symptoms on every DSQ domain, as well as the lowest levels of functioning on the majority of SF-36 subscales. Additionally, the Constantly Getting Worse group reported overextending their personal energy envelopes to significantly higher degree compared to the average patient. In short, the illness experiences of those within this subtype seem distinctly more severe compared to the majority of patients experiencing ME or CFS.
Participants that identified with the Persisting illness course subtype comprised $14.1 \%$ of the total sample. This illness course was described to them as having no changes in symptomatology. Overall, this group did not display a consistent pattern with regards to severity of symptoms and levels of functioning. On some DSQ domains, such as the Gastrointestinal, Orthostatic Intolerance, and Neuroendocrine/Circulatory, this group reported low severity and frequency of symptoms. However, on other DSQ domains and SF-36 subscales, this group ranked behind the Constantly Getting Worse group in terms of the highest levels of symptom frequency and severity and the lowest levels of functioning. Therefore, the results indicated that this group showed a moderate to high level of severity. In fact, the only significant difference between the Persisting group and the majority group (Fluctuating) was on the Vitality subscale of the SF-36, on which the Persisting group reported lower functioning. In addition, the Persisting subtype reported significantly higher levels of fatigue, as well as less available energy when compared to the Fluctuating group. However, due to the relatively few differences that occurred between these groups, future investigation is needed to further explore the distinctive illness experience of this subtype.

Participants among the Relapsing and Remitting group represented $8.5 \%$ of the total sample. This illness course was described as involving "good" periods with no symptoms, alternat- 
ing with symptomatically "bad" periods. Overall, this group reported moderate levels of symptomology and functioning when compared to other groups. However, our findings do not indicate substantial differences in experiences between those in the Relapsing and Remitting group and the Fluctuating group. The only scores which significantly differed were the Mental Health subscale scores of the SF-36, on which the Relapsing and Remitting group reported higher functioning. Because of this, it is difficult to specify a distinct symptomatic profile for this group, and future research may consider combining these two subtypes. Indeed, when we repeated all analyses with the Fluctuating and Relapsing and Remitting groups combined, we found that collapsing these subtypes did not result in noticeable changes in the overall findings. Because the approach taken here was exploratory in nature, we decided that keeping these subtypes separate for now could prove to be important for future analyses. However, further examination is needed to better understand the differences between symptomology and functioning for these illness courses.

Finally, the Constantly Improving group, comprising $1.9 \%$ of the total sample, consistently displayed a pattern of lower frequency and severity for symptoms on the DSQ and better overall functioning as demonstrated on the SF-36 subscales. Additionally, on average this group tended to expend more energy than they had available. This supports past research which suggests that some patients may be more able to overextend themselves with little consequence to their symptomology and functioning [27]. However, as only a small proportion $(n=10)$ of the present sample endorsed Constantly Improving as their illness trajectory, the conclusions that can be drawn about their illness experiences are limited. Nonetheless, the fact that there are so few participants within this subtype supports findings that suggest recovery from these debilitating illnesses is rare [28].

While the subtypes presented in this study provide promise in classifying more homogenous groups of patients, the approach taken had several limitations. First, it is difficult to assess participants' illness experiences over time when using measures completed at a single time point. Items on the DSQ ask participants to rate the frequency and severity of their symptomatology over the past 6 months. Likewise, items on the SF-36 ask participants to rate their levels of functioning on a "typical day" over the past four weeks. The format of these items proves especially problematic for assessing the experiences of those within the Fluctuating and Relapsing and Remitting groups. As the nature of these groups' illness course involves a variation in symptoms over time, when asking them to average their functioning and symptomology over time, we fail to capture a complete appreciation for the patterns of their illness.

Another limitation of the current study is that the majority of participants identified as Caucasian. This differs from the demographic distribution found in past community-based samples, which have documented higher levels of CFS diagnosis in ethnic minorities and those with lower socioeconomic status [29]. It would be important to consider whether patients from minority backgrounds differ in their illness course experiences, as studies have shown differences in patterns of symptom severity and functional status between different sociocultural samples
[30]. However, the use of multiple geographically distinct collection sites was an advantage in that it lends support for the possibility of generalizing these results over multiple recruitment settings and geographic locations. Future studies should include greater ethnic and sociocultural diversity as well as consider utilizing a longitudinal design to more accurately measure each participant's illness course. Measuring how symptoms and levels of functioning change over time would help distinguish clear patterns between subtypes and allow for further verification of the distinctiveness of these illness courses.

\section{Conclusions}

These preliminary findings demonstrate that illness course subtypes can account for significant differences in the experiences of patients with CFS and ME. The severity and frequency of symptoms, levels of functioning, and utilization of energy were shown to differ significantly between illness course subtypes. Continuing to explore and develop the illness course classification approach is an important step in furthering treatment and research for this debilitating illness. Not only will this lead to a more nuanced understanding of treatment efficacy and prognosis, but it will allow researchers to consistently categorize patients by illness subtype increasing the specificity of their research. This would likely ultimately lead to more consistent findings within the field.

\section{Acknowledgments}

This work was supported by the National Institute of Allergy and Infectious Diseases under Grant (AI105781).

\section{Declarations}

\section{Ethical Approval}

The ethical standards of the American Psychological Association were upheld throughout the study period. All procedures were approved by the DePaul University Institutional Review Board.

\section{References}

1. Fukuda K, Straus SE, Hickie I, Sharpe MC, Dobbins JG, Komaroff A. The chronic fatigue syndrome: a comprehensive approach to its definition and study. International Chronic Fatigue Syndrome Study Group. Ann Intern Med. 1994;121(12):953-959.

2. Carruthers BM, Jain AK, Meirleir, Kenny L. De Meirleir, Daniel L. Peterson, Nancy G. Klimas, et al. Myalgic encephalomyelitis/chronic fatigue syndrome: Clinical working case definition, diagnostic and treatment protocols. Journal of Chronic Fatigue Syndrome. 2011;11(1):7-115. doi: 10.1111/j.1365-2796.2011.02428.x

3. Carruthers BM., van de Sande MI, De Meirleir KL, N. G. Klimas, G. Broderick, T. Mitchell, et al. Myalgic encephalomyelitis: International Consensus Criteria. Journal of Internal Medicine. 2011;270(4):327-338. doi: 10.1111/j.1365-2796.2011.02428.x

4. IOM (Institute of Medicine). Beyond myalgic encephalomyelitis/chronic fatigue sydrome: Redefining an illness (Report Brief). Washington, DC: National Academy of Sciences. 2015. 
5. Friedberg F, Jason LA. Understanding chronic fatigue syndrome: An empirical guide to assessment and treatment. 1st ed. American Psychological Association (APA). 1998.

6. Pendergrast T, Brown A, Sunnquist M, Jantke R, Newton JL, Strand EB, et al. Housebound versus nonhousebound patients with myalgic encephalomyelitis and chronic fatigue syndrome. Chronic Illn. 2016;12(4):292-307. doi: 10.1177/1742395316644770

7. Jason LA, Sunnquist M, Brown A, Furst J, Cid M, Farietta J, et al. Factor Analysis of the DePaul Symptom Questionnaire: Identifying Core Domains. J Neurol Neurobiol. 2015;1(4). doi: 10.16966/2379-7150.114

8. Jason LA, Taylor RR, Kennedy CL, Harding ST, Song S, Johnson D, et al Subtypes of Chronic Fatigue Syndrome: A Review of Findings. Journal of Chronic Fatigue Syndrome. 2001;8(3-4),1-21. doi: 10.1300/ J092v08n03_02

9. DeLuca J, Johnson SK, Ellis SP, Natelson BH. Sudden vs gradual onset of chronic fatigue syndrome differentiates individuals on cognitive and psychiatric measures. J Psychiatr Res. 1997;31(1):83-90.

10. Levine PH. Epidemiologic advances in chronic fatigue syndrome. J Psychiatr Res. 1997;31(1):7-18.

11. Reyes M, Dobbins JG, Nisenbaum R, Subedar NS, Randall B, Reeves WC. Chronic Fatigue Syndrome Progression and Self-Defined Recovery: Evidence from the CDC Surveillance System.1999;5(1):17-27. doi: 10.1300/J092v05n01_03

12. Hill N, TierskyL, Scavalla V, Natelson B. The fluctuation and outcome of chronic fatigue syndrome (CFS) over time. Journal of Chronic Fatigue Syndrome. 1999;5:93-94.

13. Friedberg F, Dechene L, McKenzie MJ, Fontanetta R. Symptom patterns in long-duration chronic fatigue syndrome. J Psychosom Res. 2000;48(1):59-68.

14. Matthews RM, Komaroff AL. Changes in functional status in chronic fatigue syndrome over a decade. Journal of Chronic Fatigue Syndrome. 2007;14(1):33-42.

15. Joyce J, Hotopf M, Wessely S. The prognosis of chronic fatigue and chronic fatigue syndrome: a systematic review. QJM: Monthly Journal of the Association of Physicians. 1997;90(3):223-233.

16. Kidd E, Brown A, McManimen S, Jason LA, Newton JL, Strand EB. The relationship between age and illness duration in chronic fatigue syndrome. Diagnostics. Diagnostics (Basel). 2016;6(2). doi: 10.3390/diagnostics6020016.

17. Komaroff AL. Chronic fatigue syndromes: relationship to chronic viral infections. J Virol Methods. 1988;21(1-4):3-10.

18. Glaser R, Padgett DA, Litsky ML, Baiocchi RA, Yang EV, Chen M, et al. Stress-associated changes in the steady-state expression of latent Epstein-Barr virus: Implications for chronic fatigue syndrome and cancer. Brain Behav Immun. 2005;19(2):91-103. doi: 10.1016/j. bbi.2004.09.001
19. Chia JKS, Chia AY. Chronic fatigue syndrome is associated with chronic enterovirus infection of the stomach. J Clin Pathol. 2008;61(1):43-48.

20. Lublin FD, Reingold SC, Cohen JA, Cutter GR, Sørensen PS, Thompson AJ, et al. Defining the clinical course of multiple sclerosis the 2013 revisions. Neurology. 2014;83(3):278-286. doi: 10.1212/ WNL.0000000000000560.

21. Ware JE, Sherbourne CD. The MOS 36-item short-form health survey (SF-36). I. Conceptual framework and item selection. Med Care. 1992;30(6):473-483.

22. McHorney CA, Ware JE, Lu JFR, Sherbourne CD. The MOS 36-item short-form healthsurvey (SF-36): III. tests of data quality, scaling assumptions, and reliability across diverse datient groups. Med Care. 1994;32(1):40-66.

23. Jason LA, McManimen S, Sunnquist M, Brown A, Furst J, Newton, JL, et al. Case definitions integrating empiric and consensus perspectives. Fatigue: Biomedicine, Health \& Behavior. 2016;4(1):1-23.

24. Jason LA, So S, Brown AA, Sunnquist M, Evans M. Test-retest reliability of the DePaul Symptom Questionnaire. Fatigue: Biomedicine, Health \& Behavior. 2015;3(1):16-32. doi: 10.1080/21641846.2014.978110

25. Brown AA, Jason LA. Validating a measure of myalgic encephalomyelitis/chronic fatigue syndrome symptomatology. Fatigue: Biomedicine, Health \& Behavior. 2014;2(3):132-152. doi: $10.1080 / 21641846.2014 .928014$

26. King C, Jason LA, Frankenberry E, \& Jordan K. Think inside the enve lope. CFIDS Chronicle.1997;10-14.

27. Brown AA, Evans MA, Nev Jones, Jason LA. Examining the energy envelope and associated symptom patterns in chronic fatigue syndrome: does coping matter? Chronic Illness. 2013;9(4):302-311. doi: $10.1177 / 1742395313478220$

28. Cairns R, Hotopf M. A systematic review describing the prognosis of chronic fatigue syndrome. Occupational Medicine (Oxford, England). 2005;55(1):20-31. doi: 10.1093/occmed/kqi013

29. Jason LA, Taylor R, Wagner L, Holden Jay, Ferrari Joseph R., Plioplys Audrius V., et al. Estimating rates of chronic fatigue syndrome from a community-based sample: A pilot study. American Journal of Community Psychology. 1995;23(4):557-568. doi: 10.1007/BF02506968

30. Zdunek M, Jason LA, Evans M, Jantke R, Newton JL. A cross cultural comparison of disability and symptomatology associated with CFS. International Journal of Psychology and Behavioral Sciences. 2015;5(2):98-107. doi: 10.5923/j.ijpbs.20150502.07 